\title{
In Silico Screening, Alanine Mutation, and DFT Approaches for Identification of NS2B/NS3 Protease Inhibitors
}

\author{
R. Balajee, ${ }^{1}$ V. Srinivasadesikan, ${ }^{2}$ M. Sakthivadivel, ${ }^{3}$ and P. Gunasekaran ${ }^{3}$ \\ ${ }^{1}$ Medicinal Chemistry Group, Institute of Chemistry of Sao Carlos, University of Sao Paulo, Sao Carlos, Brazil \\ ${ }^{2}$ Department of Applied Chemistry, National Chiao Tung University, Hsinchu, Taiwan \\ ${ }^{3}$ King Institute of Preventive Medicine, Guindy, Chennai, India \\ Correspondence should be addressed to R. Balajee; rbalajeebio@gmail.com
}

Received 26 September 2015; Revised 13 December 2015; Accepted 15 December 2015

Academic Editor: Roberta Chiaraluce

Copyright (c) 2016 R. Balajee et al. This is an open access article distributed under the Creative Commons Attribution License, which permits unrestricted use, distribution, and reproduction in any medium, provided the original work is properly cited.

\begin{abstract}
To identify the ligand that binds to a target protein with high affinity is a nontrivial task in computer-assisted approaches. Antiviral drugs have been identified for NS2B/NS3 protease enzyme on the mechanism to cleave the viral protein using the computational tools. The consequence of the molecular docking, free energy calculations, and simulation protocols explores the better ligand. It provides in-depth structural insights with the catalytic triad of His51, Asp75, Ser135, and Gly133. The MD simulation was employed here to predict the stability of the complex. The alanine mutation has been performed and its stability was monitored by using the molecular dynamics simulation. The minimal RMSD value suggests that the derived complexes are close to equilibrium. The DFT outcome reveals that the HOMO-LUMO gap of Ligand19 is $2.86 \mathrm{kcal} / \mathrm{mol}$. Among the considered ligands, Ligand19 shows the lowest gap and it is suggested that the HOMO of Ligand19 may transfer the electrons to the LUMO in the active regions. The calculated binding energy of Ligand19 using the DFT method is in good agreement with the docking studies. The pharmacological activity of ligand was performed and satisfies Lipinski rule of 5. Moreover, the computational results are compared with the available $\mathrm{IC}_{50}$ values of experimental results.
\end{abstract}

\section{Introduction}

Dengue virus (DV, DENV 1-4) belongs to Flaviviridae family; infection regardless of its serotypes is transmitted from person to person by the female leads of Aedes aegypti or Aedes albopictus mosquitoes in the domestic environment and causes a serious public health issues across the globe $[1,2]$. Recently, two thousand cases were reported in Portugal and many cases were detected in European part and many cases in Asia [3]. It is one of the most prevalent arthropodborne viral diseases in terms of morbidity and mortality [4].

An integral membrane protein of NS2B and NS3 (NS2B/NS3) in the serine protease enzyme is essential for viral replication [5]. The structural insight shows that NS3 belongs to a second larger protein and contains the catalytic triad and is colocalized within distinct paracrystalline of convoluted membranes [6]. It is termed as crucial because it is being matured with the presence of cofactor NS2B and enables the polyprotein posttranslationally in endoplasmic reticulum $[7,8]$. The processing occurs in $\mathrm{P} 1$ and $\mathrm{P} 2$ positions, where P2 position carries Lys-Arg, Arg-Arg, and Arg-Lys and oftenly P1 position carries Gln-Arg followed by Gly, Ala, or Ser. Therefore, the biologically active viral protease of NS2B/NS3 complex is termed as heterodimeric [9]. The hydrophobic regions are associated with the polyprotein precursor, which regulates the protease sensitive sites in the optimal context of cis- and trans-cleavages. Therefore, the biologically active viral protease of NS2B/NS3 complex is termed as heterodimeric [9].

Earlier reports states that the various inhibitors from the different sources are analyzed using in vitro and in silico approaches to determine their biological activity and binding modes with close proximity residues. The active site was detected in the major surface of the C-terminal domain region and substrate binds to the polyprotein sequences to carry out the cleavage process [10]. The series of inhibitors like panduratin, hydroxypanduratin, and so forth have been explored from the molecular docking analysis. The key 


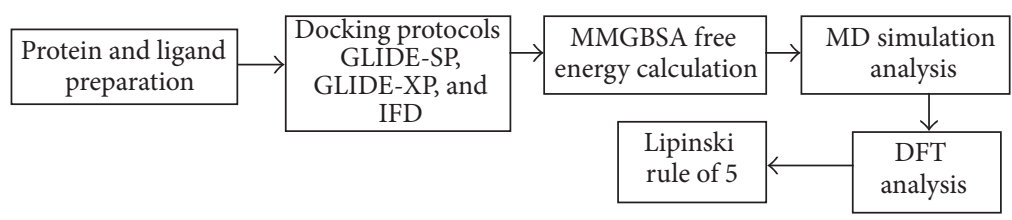

FIGURE 1: Schematic representation of proposed research.

residue responsible for interaction between the protein and inhibitors was established [11]. The alanine substitution detects the precleavage at NS2B/NS3 complex and may cause the proteolytic activity to decline [12]. The mutation in complex causes the proteolytic activity to decline [13]. Hence, the proposed complex is analyzed to be a promising target in the search for drugs against DV.

Frontiers in the drug discovery research challenges the pharmaceutical industries [14]. So, the scientific community is taking more efforts to discover the therapeutic target towards the NS2B/NS3 proteins. Currently, the work is designed to identify the novel potential inhibitor for the NS2/NS3 protease enzyme for dengue virus. The study was observed from various computational protocols like molecular docking, molecular dynamics simulation, free energy calculations, and DFT approaches and the flow chart is shown in Figure 1. MD simulations were performed to determine the stability and dynamical changes of predicted binding conformation and analysis extended between Wild Type (WT) and mutated consequences. A MM-GB/SA analysis was carried out to calculate the binding free energies of the complex and to determine the characteristics of drug-like molecule towards the NS2/NS3 protease enzyme. Moreover, the state-of-the-art density functional theory (DFT) investigations have also been carried out at M06 [15] level with the 6-31g(d) basis set to better understand the hydrogen bonding strengths, binding energy, and the interaction sites of amino acids with the ligands at molecular level. The properties of drug-like molecule like hydrogen bond, physicochemical parameters of Lipinski rule of 5 (RO5) [16], and the results from this study will be highly useful for further investigations.

\section{Materials and Methods}

The Schrodinger molecular modelling suite was employed to carry out the molecular docking, dynamics simulation analysis, and free energy estimations. To determine the hydrogen bonding strengths, molecular orbitals (HOMOLUMO), electrostatic potential (ESP), and binding energy between ligand selected amino acids the density functional theory calculations have been carried out by using Gaussian 09 program [17]. Due to the computational limitation we have considered only two to three key amino acids which are interacted with the ligands for the DFT study. And we have taken four ligands for the DFT investigation which have shown the higher binding energy and scoring function in the docking studies. The further DFT study has been carried out to understand the key amino acids in the binding pocket interacting with the ligands at molecular level. The geometries are optimized at M06/6-31g(d) level of theory in gas phase. Molecular orbitals are visualized using Gauss view 5.0.8. The binding energies are calculated using the following formula:

$$
\begin{aligned}
\text { Binding Energy }(\mathrm{BE})= & E_{\text {Ligand-Amino acids }} \\
& -\left(E_{\text {Ligand }}+E_{\text {Amino acids }}\right) .
\end{aligned}
$$

And the electrostatic potential (ESP) calculations have been carried out using the fchk and pop, regular key words in Gaussian 09.

2.1. Ligand Background. The binding affinity between the receptor and inhibitor determines the biological activity; it was influenced by geometrical positions and steric and physical properties. The distinct of structures was explored and leads to the large set of biological diversities. For the investigation, the ligands were obtained from Pubchem/Drugbank and Chemdiv as shown in Table 1 [10]. The structure of these inhibitors along with binding energies and $\mathrm{IC}_{50}$ values was given in Table 1.

2.2. Preparation Methodologies. A diverse set of ligands were obtained from PubChem [18], DrugBank [19], and Chemical Diversity, USA [20, 21]. Each ligand was subjected to a minimization with the OPLS2005 force field to eliminate the steric clashes of bond length and angles in the crystal structure and prepared with $\mathrm{pH} 7.0$ using LigPrep module [22, 23]. The structural coordinates of dengue virus NS2B/NS3 protease were extracted from the Protein Data Bank (PDB ID: 2fom) [24] and structure was minimized using IMPACT module with steepest descent gradient algorithm for about 100 cycles [25]. The structure was optimized using protein preparation wizard in a similar fashion [26].

2.3. Docking Protocol. GLIDE parameters were applied with VdW scaling of 0.8 and partial cut-off of 0.15 was implemented to soften the potential for nonpolar sites and no constraints were specified. The grid box was generated according to the residues suggested with the VdW scaling factor of 0.8 and partial cut-off of 0.15 [27]. The ligands were docked using the "standard precision" (GLIDE-SP) and "extra precision" (GLIDE-XP) [28]. Firstly, the ligands were screened using GLIDE-SP and the GLIDE-XP approach was executed to ensure the good score and poses. The outcome was generated according to the GLIDE Energy and interactions [29]. The least energy values were selected for further analysis. 
TABLE 1: GLIDE-XP energies ( $\mathrm{kcal} / \mathrm{mol})$ of selected 20 ligands with $\mathrm{IC}_{50}$ values.

\begin{tabular}{lcccc}
\hline Sl. number & Ligand & Source & Energy & $\mathrm{IC}_{50}(\mu \mathrm{M})$ \\
\hline 1 & Ligand1 & PubChem & -34.18 & 3.0 \\
2 & Ligand2 & PubChem & -36.51 & 1.29 \\
3 & Ligand3 & PubChem & -43.10 & 1.29 \\
4 & Ligand4 & PubChem & -46.75 & 1.29 \\
5 & Ligand5 & PubChem & -38.16 & 1.29 \\
6 & Ligand6 & PubChem & -44.67 & 33.89 \\
7 & Ligand7 & PubChem & -45.83 & 0.1 \\
8 & Ligand8 & ChemDiv & -48.40 & 15.6 \\
9 & Ligand9 & ChemDiv & -44.21 & 86.7 \\
10 & Ligand10 & ChemDiv & -31.34 & 32.2 \\
11 & Ligand11 & ChemDiv & -41.67 & 12.5 \\
12 & Ligand12 & ChemDiv & -39.14 & 3.9 \\
13 & Ligand13 & ChemDiv & -39.09 & 26.4 \\
14 & Ligand14 & ChemDiv & -51.31 & 75.3 \\
15 & Ligand15 & PubChem & -42.52 & 0.12 \\
16 & Ligand16 & PubChem & -31.57 & 4.7 \\
17 & Ligand17 & PubChem & -35.18 & 1.08 \\
18 & Ligand18 & PubChem & -36.41 & 0.009 \\
19 & Ligand19 & PubChem/DrugBank & -41.00 & 1.37 \\
20 & Ligand20 & PubChem/DrugBank & -41.16 & 1.37 \\
\hline
\end{tabular}

\section{Induced Fit Docking (IFD) Protocol}

The outcome of GLIDE-XP was considered to perform the IFD protocol and this is termed as second phase. This is considered as a flexible protein with flexible ligand. The resulting ligands were docked individually using the following steps: (a) the receptor was defined with the aforementioned active site residues and (b) in the initial GLIDE docking stage soften potential docking with the van der Waals radii scaling of 0.50 [30] for the protein was performed to retain the maximum number of 5 poses per ligand.

\section{MMGBSA Protocol}

The Prime-MM/GBSA protocol was employed to predict the free energy of binding complexes. The complex was minimized with the OPLS-2005 Force Field [31] Prime which uses the VSGB model employing a surface representation of the Solvent Accessible Surface Area [32].

\section{Dynamics Calculations}

MD calculations were performed using Desmond version 2.0, Schrodinger LLC. The best outcome of IFD was initiated with TIP3P water model and orthorhombic box buffer size of $10 \AA$ and the system was neutralized with $0.15 \mathrm{M}$ using $\mathrm{Na}^{+}$ions. Force field parameters of the protein-ligand systems are assigned with OPLS-2005, constrained using the SHAKE algorithm with the period boundary conditions (PBC), and electrostatic interactions were applied using the Particle Mesh Ewald (PME) method [33]. For the dynamics, a multistep
TABLE 2: Induced Fit Docking results.

\begin{tabular}{lcc}
\hline Sl. number & Ligand & Energy \\
\hline 1 & Ligand7 & -49.17 \\
2 & Ligand8 & -62.71 \\
3 & Ligand19 (pose 1) & -47.00 \\
4 & Ligand19 (pose 2) & -64.16 \\
\hline
\end{tabular}

RESPA integration algorithm was used throughout $1.2 \mathrm{fs}$. The relaxation was followed by 10 ns production run in the NPT ensemble with temperature $300 \mathrm{~K}$, thermostat relaxation time $1.0 \mathrm{ps}$, and barostat relaxation time $2.0 \mathrm{ps}$ which was performed for each system using a Nose-Hoover thermostat and Martyna-Tobias-Klein barostat and prepared with similar fashion for the period of $10 \mathrm{~ns}$ [34].

5.1. Mutational Analysis. The alanine was substituted with the following residues Leu115, Asp129, Gly133, Thr134, Ser163, and Ile165 which are suggested in order to determine the protein stability [35]. The docking and simulation interpretation was performed with the aforementioned methodologies.

\section{Results and Discussions}

6.1. Docking Analysis. The region of NS2B protein associated with NS3 protein has greater impact to enhance their activity [36]. The screened ligands (Figure 2) categorized according to the energy values reported in Table 1 and the respective hydrogen bond interactions are shown in Figure 3. The result reveals that Ligand7, Ligand8, Ligand14, and Ligand19 are much closer towards active site. These ligands are further analyzed with Induced Fit Docking approach.

6.2. Induced Fit Docking. Induced Fit Docking (IFD) approach was aimed to detect the conformational changes of the protein and to study the characteristics of the ligands. The analysis was performed from the outcome of the GLIDE-XP. The IFD result reveals the better poses in contrast to the more poses generated for the following ligands: Ligand7, Ligand8, and Ligand19, and the least energy values $-49.17,-62.71$, and -64.16 , respectively, reported in Table 2. Ligand19 of pose 2 has a better interaction with the catalytic site given in Table 3 and exhibits realistic energy value to have a property of drug-like molecule. The outcome of IFD was advanced to estimate the binding affinity in the free energy state.

\section{MM-GBSA Docking}

The calculated free energy complex ranges from -53 to $-74 \mathrm{Kcal} / \mathrm{mol}$ shown in Table 4 . According to the MMGBSA results, the major favourable contributions to the ligand binding are $\operatorname{VdW}\left(\Delta G_{\mathrm{vdw}}\right)$ and solvation terms $\left(\Delta G_{\mathrm{sol}}\right)$. These results provide more significance towards the binding affinity depicted in Figure 4 . Therefore, this study describes that $G_{\text {solv }}$ is an important factor to steer the force for ligand binding.

The docking protocol was evaluated in order to identify the distance between the active site residues and ligands 
<smiles>CNC(C)c1ccc2cccnc2c1O</smiles><smiles>Oc1c(C(NC2=NC3C=CC=CC3S2)c2ccccc2)ccc2cccnc12</smiles><smiles>Oc1ccccc1C(NC1=NC2C=CC=CC2S1)c1ccc2cccnc2c1O</smiles><smiles>Oc1cccc(C(Nc2nccs2)c2ccc3cccnc3c2O)c1</smiles><smiles>C[C@@H]1O[C@H](O[C@@H]2[C@@H](O)C[C@@H](O)O[C@H]2[O-])C[C@H]([O-])[C@@H]1O</smiles>

7

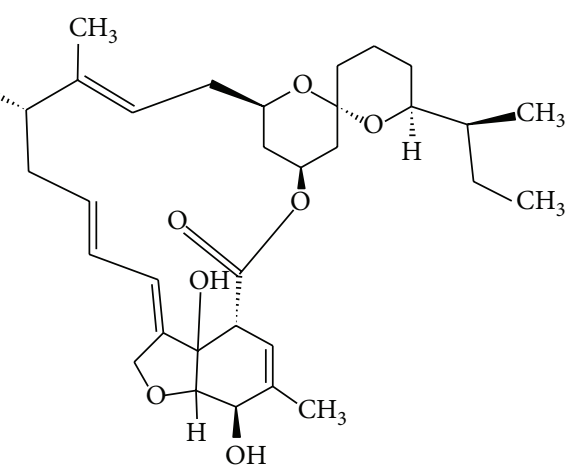<smiles>O=S(=O)(c1ccc(-c2csc(N3N=C(c4cccs4)CC3c3ccc(F)cc3)n2)cc1)N1CCCCC1</smiles><smiles>CCN(CC)CCn1c(NC(=O)c2ccc(Cn3nc([N+](=O)[O-])c(Cl)c3C)o2)nc2ccccc21</smiles><smiles>Cc1nnc2c(NC3=C=CC=C(C(=O)O)C3)nc3c(n12)=CC(C(=O)c1ccccc1)CC=3</smiles>

FIGURE 2: Continued. 
11<smiles>CC(=O)N1N=C(c2cc(-c3ccccc3)nc3ccccc23)OC1c1ccc(C(=O)O)cc1</smiles>

12<smiles>Cc1cccc(C)c1-n1c(SCc2cc(=O)n3cc(Cl)ccc3n2)nc2c(sc(=S)n2-c2c(C)cccc2C)c1=O</smiles>

15<smiles>O=C(O)CCCNc1cc(N2CCCCCC2)c2noc3c2c1C(=O)c1ccccc1-3</smiles><smiles>C[C@@H]1OCC[C@H]([O-])[C@H]1O</smiles><smiles>CC1CCCCC1</smiles>

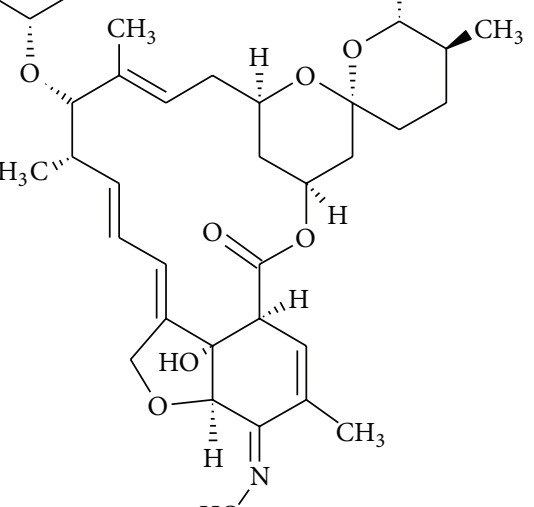

$\mathrm{HO}^{\prime}$<smiles>CCC(C)(CC(C)(C)C)c1ccc(OCCOCC[N+](C)(C)Cc2ccccc2)cc1</smiles>

17<smiles>CCCCC(CC)CNC(=N)NC(=N)NCCCCCCNC(=N)NC(=N)NCC(CC)CCCC</smiles>

Figure 2: Continued. 


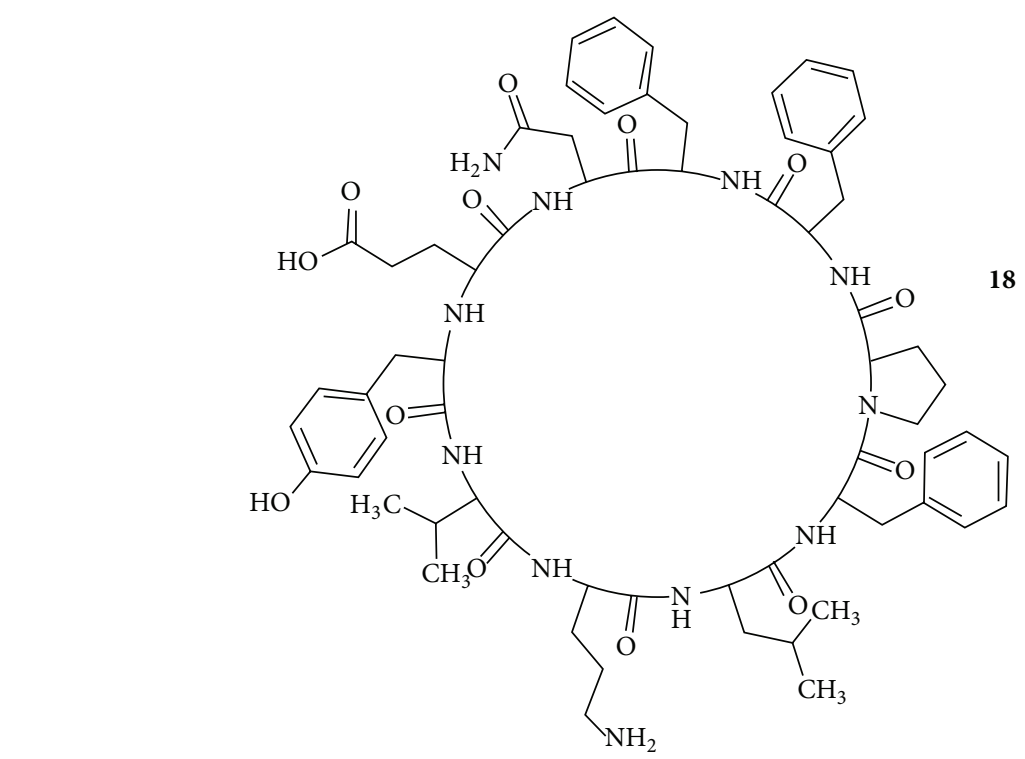

19<smiles>CCC(=O)C(=O)c1cc(/C=N/NC2=Nc3ccccc3C2)c(O)c(CNNc2nc3ccccc3[nH]2)c1</smiles>

20<smiles>O=C(N/N=C/Cc1ccccc1)c1cc(/C=N/Nc2cccc(Br)c2)nc2ccccc12</smiles>

FIGURE 2: Top ranked 20 ligand structures. Ligand numbers only provided near to each ligand structure.

TABLE 3: The best poses and their hydrogen bond distance in $\AA$.

\begin{tabular}{|c|c|c|}
\hline Ligands & Hydrogen bond interactions & Distance $(\AA)$ \\
\hline \multirow{2}{*}{ Ligand7 } & Gly151 O ‥H-O (Lig) & 2.29 \\
\hline & Ile36 O $\cdots$ H-O (Lig) & 2.00 \\
\hline \multirow{3}{*}{ Ligand $\mathbf{8}$} & Lig N-H...O Phel30 & 1.72 \\
\hline & Tyr150 O $\cdots$ H-O Lig & 2.16 \\
\hline & 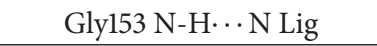 & 2.41 \\
\hline \multirow{3}{*}{ Ligand19-pose 1} & Lig N-H $\cdots$ O Phel30 & 2.09 \\
\hline & 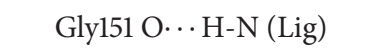 & 2.03 \\
\hline & Lig N-H $\cdots$ O Asp75 & 1.81 \\
\hline \multirow{5}{*}{ Ligand19-pose 2} & Gly151 O $\cdots$ H-O Lig & 2.03 \\
\hline & Lig N-H $\cdots$ O Gly153 & 2.48 \\
\hline & Lig N-H. O Asp75 & 2.43 \\
\hline & Lig N-H $\cdots$ H Asp75 & 2.44 \\
\hline & Lig N-H... O Phe130 & 2.06 \\
\hline
\end{tabular}

targeted between $1.7 \AA$ and $2.5 \AA$. The following insights were observed from the earlier report of Murthy et al. and Kee et al. [37, 38]; the Gly133 and Ser135 were actively contributed in oxyanion hole and the catalytic triad was generated with the residues of His51, Asp75, and Ser135 which incorporated in the active site regions. In this context, the interactions are being considered as main criteria; Ligand19 seems to be a potential and interacted with the least distance to the following residues: Asp75, Gly151, and Gly153, when compared with existing ligands like panduratin and hydroxypanduratin [11] shown in Table 5. It shows a better interaction with respect to the distances with existing residues as well as established interaction with Phe130 provided as the stable contact shown in Figure 4.

The strong interactions $\mathrm{N}-\mathrm{H} \cdots \mathrm{O}$ and $\mathrm{O}-\mathrm{H} \cdots \mathrm{O}$ are more crucial towards the protein-ligand binding [39]. At this point, the reported ligand having the direct contact about the distance of $\sim 2.4 \AA$ with the active regions, especially carboxyl oxygen group of Phe130, is targeted towards amine group 
TABLE 4: Binding free energies derived from MM-GBSA calculations using single protein-ligand complexes (unit: $\mathrm{kcal} / \mathrm{mol}$ ).

\begin{tabular}{lcccccr}
\hline Sl. number & Ligand & $\Delta G_{\text {coul }}$ & $\Delta G$ & $\Delta G_{\text {sol }}$ & $\Delta G_{\text {lipo }}$ & $\Delta G_{\text {bind }}$ \\
\hline 1 & Ligand7 & -20.706 & -42.822 & 6.453 & 24.162 & -20.698 \\
2 & Ligand8 & -24.938 & -37.107 & 2.206 & 16.482 & -53.611 \\
3 & Ligand19 & -26.933 & -42.448 & 1.552 & 27.567 & -34.798 \\
\hline
\end{tabular}

TABLE 5: Comparison with existing ligands.

\begin{tabular}{lccccc}
\hline Residues & 4-Hydroxypanduratin A & Panduratin A & Ligand 19 & Ligand 8 & Ligand 7 \\
\hline Ile36 & & & & $\sqrt{ }$ \\
His51 & $\sqrt{ }$ & & $\sqrt{ }$ & \\
Asp75 & & $\sqrt{ }$ & & \\
Gly133 & $\sqrt{ }$ & $\sqrt{ }$ & $\sqrt{ }$ & $\sqrt{ }$ \\
Phe130 & $\sqrt{ }$ & $\sqrt{ }$ & $\sqrt{ }$ & \\
Gly151 & & & $\sqrt{ }$ & \\
Gly153 & & & & \\
\hline
\end{tabular}
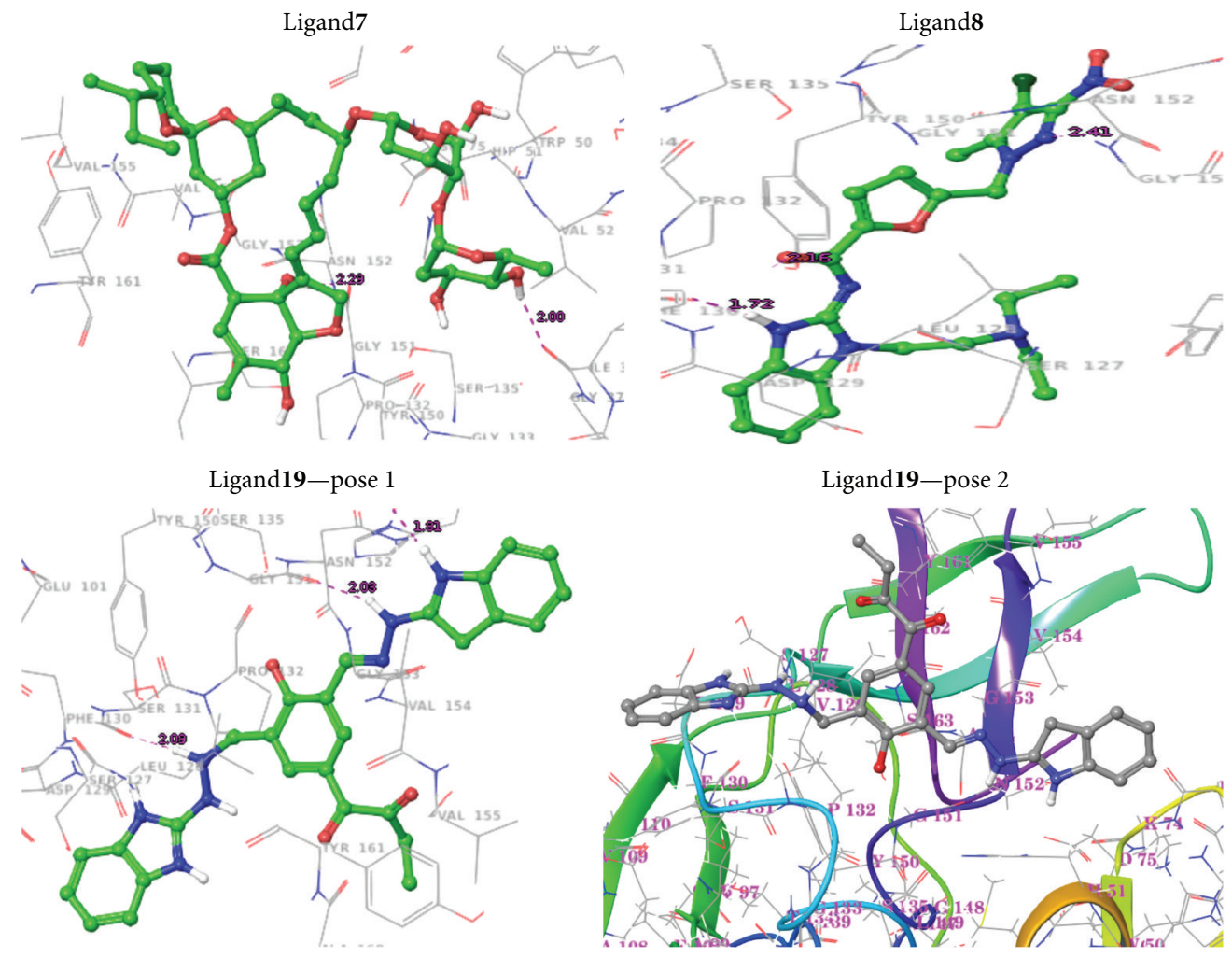

FIGURE 3: Interactions of ligand-protein complexes.

of the ligand as it may be involved in electrostatic forces. Gly151 and Asp75 residue of carboxylic oxygen group have a reasonable contact with amine group of the ligand. In another site, Gly153 of carboxyl group has an interaction with the amine group of the ligand. The results reveal the stability by displaying more contacts in the form of $\mathrm{N}-\mathrm{H} \cdots \mathrm{O}$. Pertaining to this investigation, the ligand resided in the binding pocket with the greater impact on interactions to play the role of a drug-like character and makes the oxyanion hole and the catalytic triad more active. The identified ligand molecule has a good agreement with the earlier reports [11,39] and can be considered as an inhibitor against the dengue virus NS3 protease.

Our results are in good agreement with the previous report which shows that the aforementioned residues His51, Asp75, Gly133, Ser135, Gly151, Asn152, and Gly153 are located 


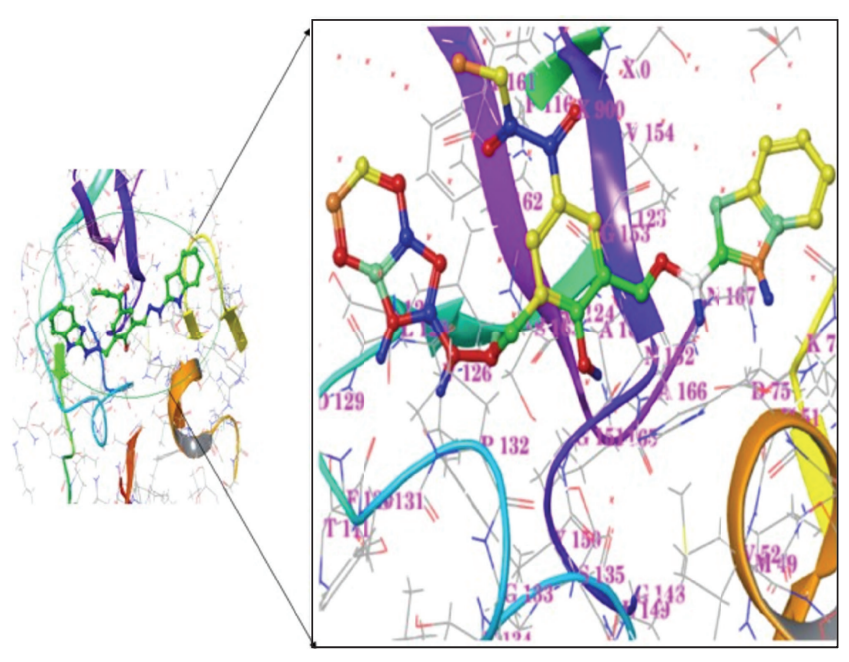

FIGURE 4: A close-up view representation of docked complexes from MM-GBSA analysis. The ligand maintains the direct contact with Phe130, Gly153, Gly151, and Asp75 indicated in the circled regions and zoomed on with their nearest residues indicated.

in the active site regions and are crucial to the ligand interactions [21, 40]. Moreover, Ser131 has been contributed in VdW interactions revealed from the docking analysis.

\section{Simulation Analysis}

Figure 5 represents the simulation analysis of the docked complex which is relatively consistent during $10 \mathrm{~ns}$ simulation run observed from the RMSD values. The hydrogen bond interactions and close proximity residues stabilized the complex, which results in the minimal RMSDs. The stretch of the RMSD peak fluctuates about $0.5 \AA$ and is boosted immediately to find its convergence after the slight fluctuations. The period between 7 and $10 \mathrm{~ns}$ seems to be crucial over stability and the fluctuation occurring at $8.5 \mathrm{~A}$ does not have a remarkable impact over the stability. Hence, this molecular dynamics simulation shows the significance towards the protein-ligand complex.

\section{Alanine Screening}

Alanine Screening-Binding Analysis. This hypothesis explored the mutational action of alanine in the Wild Type (WT) protein with the following residues: Leu115, Asp129, Gly133, Thr134, Ser163, and Ile165. The best docked ligands, Ligand08 and Ligand19, were screened with the mutated protein. Their interactions with the residues remain the same as represented in Figures 6(a) and 6(b).

Salaemae et al. [41] and Robin et al. [42] suggested that the residue Gly133 is a part of conserved motif in NS3 sequences and plays a critical role in oxyanion hole in order to ligand bind. The docking analysis reveals that alanine substitution of Gly133 residue disappears from the closer contact and the rate of the chemical reaction may be degraded [41]. The analysis of residues S163A and I165A has shown their distinguishable significance by changing their conformations

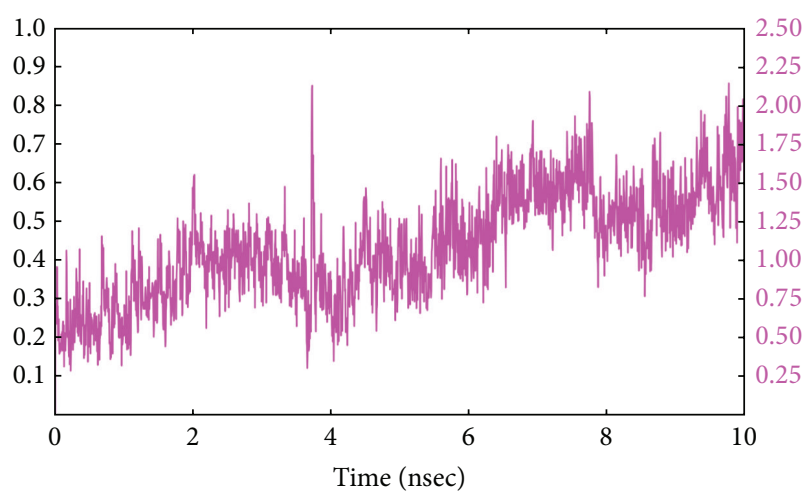

Figure 5: Simulation analysis of NS2/NS3B protease with Ligand19.

and it may also affect the enzymatic activity [43]. The side chain of His51, chain of His51, a positive charge stabilized by the cyclopentyl ring of Ligand 08 and Ligand19, was strongly supported by the carboxyl group Asp75 by obtaining the $\pi$ $\pi$ interactions. Further, Gly151 has a crucial role in form of stability from two aspects: (a) strengthening the tetrahedral transition state towards Ser135 during the substrate cleavage and (b) sustaining the stability of the protease fold [35]. Apart from the aforementioned residues the Leu115, Asp129, and Thr134 have not been involved in the drastic conformational change [41].

MD simulation analysis which provides an evidence of having a wide change between the WT and mutated proteins is represented in Figure 7(a). The WT protein has initiated their activity within a short time and shown the constant stability of RMSD values between 2.8 and $3.2 \AA$. The plateau has shown sharp fluctuation at $7.5 \mathrm{~ns}$ and it was converged at the end, whereas in mutated protein most of the residues settled between 1.5 and $3.0 \AA$ and some of the residues have shown a drastic change by specifying the plateau peaked up to $12 \AA$ which is shown in Figure 7(b). This indicates that alanine has changed the protein conformation and may disrupt the stability [44].

Our finding shows that dengue virus NS2B/NS3 protease is essentially in agreement with previous data; the molecular simulation studies confirmed that WT protein seems to be stable and mutated protein leads to a change in conformations due to alanine substitution $[43,44]$. It demonstrates that alanine substitution did not produce any remarkable effect on proteolytic cleavage [45]. The study clearly indicates the outcome of binding affinity and the simulation analysis of the mutated protein could not be able to carry out further investigations. We believe that the details provided about the designed molecule with Wild Type protein may be sufficient to execute further experimental investigations.

\section{DFT Analysis}

The DFT calculations have been carried out for the ligands which have obtained the large scoring in the docking studies. For the DFT studies maximum one to three amino acids have been considered which are shown to be interacting with 


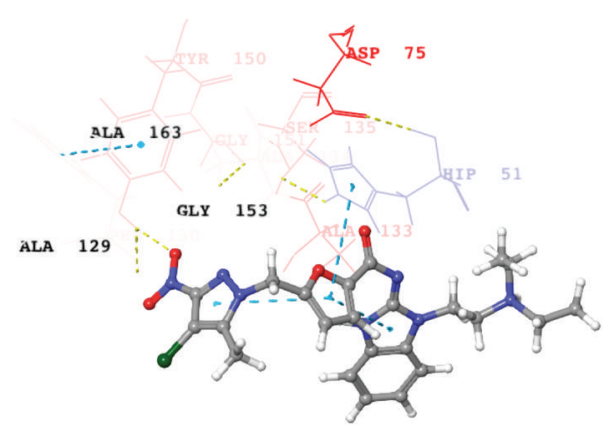

(a)

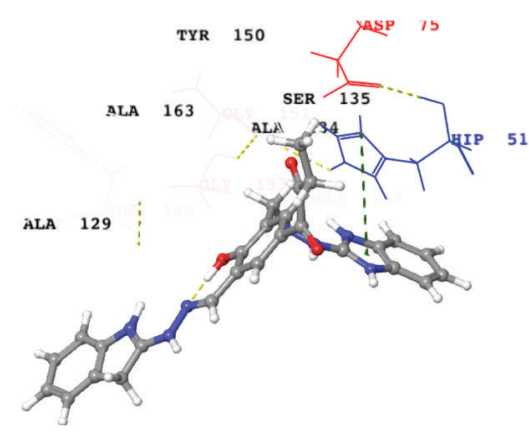

(b)

FIGURE 6: Interaction of ligands with mutated proteins: (a) Ligand08 and (b) Ligand19.

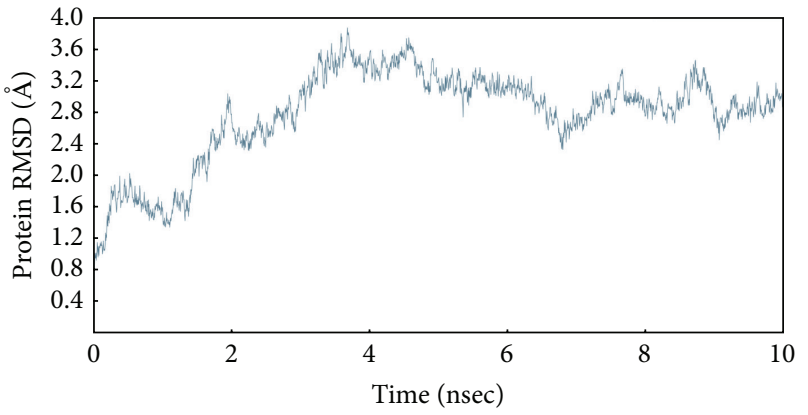

(a)

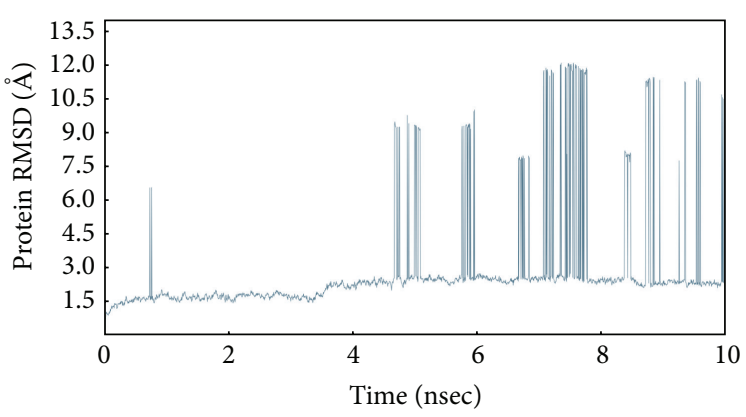

(b)

Figure 7: MD simulation of (a) WT protein and (b) alanine mutated protein.

TABLE 6: The binding energies are calculated at M06/6-31g(d) level of theory in gas phase for the ligand-amino acid complexes.

\begin{tabular}{lc}
\hline System & Binding energy $(\mathrm{kcal} / \mathrm{mol})$ \\
\hline Ligand19-Gly-Asp-Phe & -69.39 \\
7-Ile-Gly & -25.11 \\
8-Phe-Tyr & -49.09 \\
20-Gly & -8.63 \\
\hline
\end{tabular}

the ligands observed in the docking studies to determine the hydrogen bonding strengths. The calculated binding energies for the ligands, Ligand19, Ligand8, Ligand7, and Ligand20, are $-69.39,-49.09,-25.11$, and $-8.63 \mathrm{kcal} / \mathrm{mol}$, respectively. The $\mathrm{N}-\mathrm{H} \cdots \mathrm{O}, \mathrm{O}-\mathrm{H} \cdots \mathrm{N}$, and $\mathrm{O}-\mathrm{H} \cdots \mathrm{O}$ type of hydrogen bonds have been observed in the ligand(s)-amino acids complexes. All the hydrogen bond lengths are observed to be around $2.0 \AA$ which shows the stability of the complexes (Table 6). The optimized structure of Ligand19 has been shown in Figure 8. From Figure 9, it has been observed that the 2-oxobutanoyl group in Ligand19 has not participated in the amino acid interactions. In Figure 10, the frontier molecular orbital diagrams, the HOMO structure of Ligand19 shows that the electrons are localized on the central part of the ligand and are not localized on the 2-oxobutanoyl group. The LUMO structure shows that the electrons are localized at phenol and 2-oxobutanoyl groups only. From the HOMO and
TABle 7: Frontier Molecular Orbital Energies of optimized structures of Ligands19, 7, 8, and 20 at M06/6-31g(d) level in gas phase. Highest Occupies Molecular Orbital (HOMO) ${ }^{\mathrm{a}}$, Lowest Unoccupied Molecular Orbital (LUMO) ${ }^{\mathrm{b}}$, and HOMO-LUMO gap (HLG) ${ }^{\mathrm{c}}$.

\begin{tabular}{lccc}
\hline System & $\mathrm{HOMO}^{\mathrm{a}}(\mathrm{eV})$ & $\mathrm{LUMO}^{\mathrm{b}}(\mathrm{eV})$ & $\mathrm{HLG}^{\mathrm{c}}(\mathrm{kcal} / \mathrm{mol})$ \\
\hline Ligand19 & -0.199 & -0.0751 & 2.86 \\
Ligand7 & -0.2334 & -0.0299 & 4.69 \\
Ligand8 & -0.2287 & -0.0656 & 3.76 \\
Ligand20 & -0.2192 & -0.0707 & 3.42 \\
\hline
\end{tabular}

LUMO structures it can be concluded that the indole, phenol, and benzimidazole units in Ligand19 are readily available to donate the electrons to the interacting groups of amino acids in the binding site of an enzyme. The HOMO results show that Ligand19 has the highest HOMO level energy among the ligands. The results of DFT studies are in good agreement with the docking results and show that Ligand19 is the best inhibitor in the study. The HOMO-LUMO gap, in Ligand19, has been observed to be $2.86 \mathrm{kcal} / \mathrm{mol}$. The lowest HOMOLUMO gap in Ligand19 among the considered ligands is showing that HOMO of the inhibitor (Ligand19) may transfer its electrons to less energy, LUMO, of amino acids residues in the active site of an enzyme. The HOMO-LUMO gaps for the other ligands like Ligand20, Ligand8, and Ligand7 have been observed to be $3.42,3.75$, and $4.69 \mathrm{kcal} / \mathrm{mol}$, respectively, given in Table 7. 


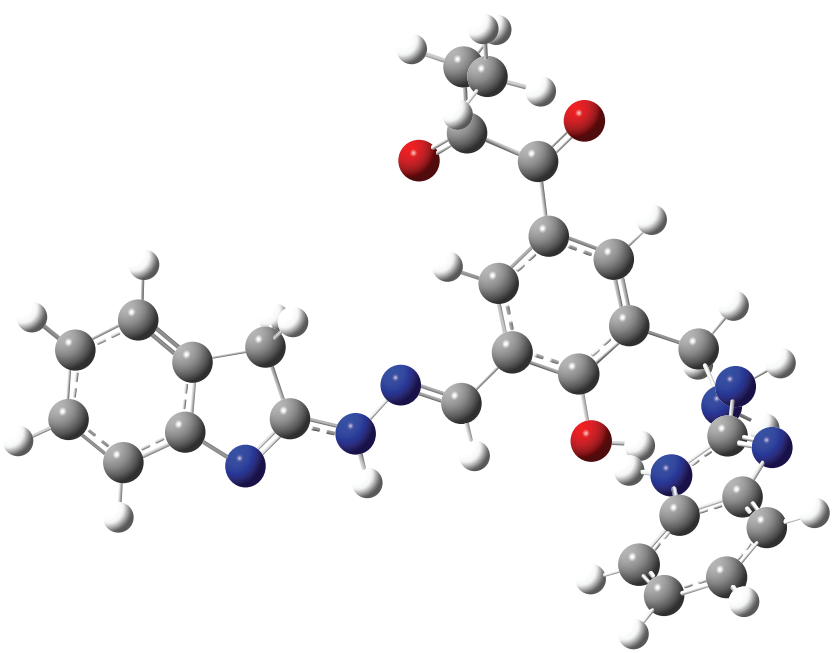

FIGURE 8: Most stable structure of Ligand19 in the gas phase optimized at M06/6-31g(d) level in Gaussian 09.

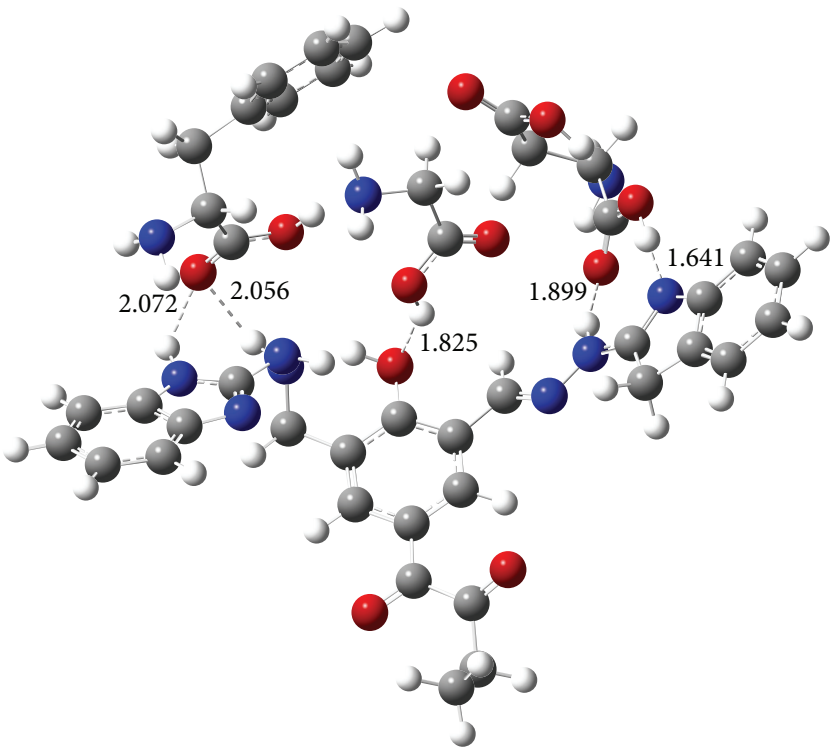

FIGURE 9: Optimized structure of Ligand19-amino acids at M06/6-31g(d) level in gas phase. The hydrogen bond lengths ( $\AA$ ) are noted.

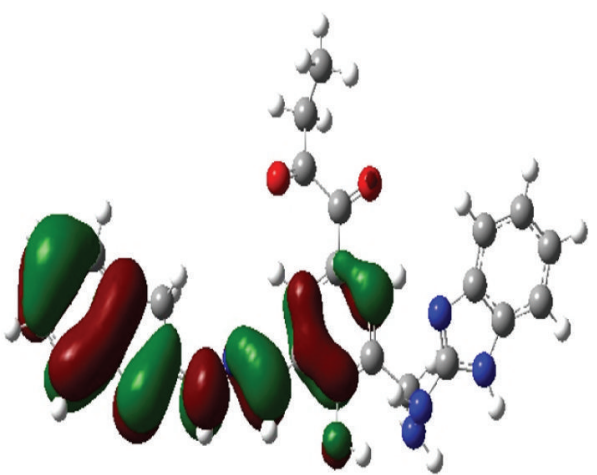

(a)

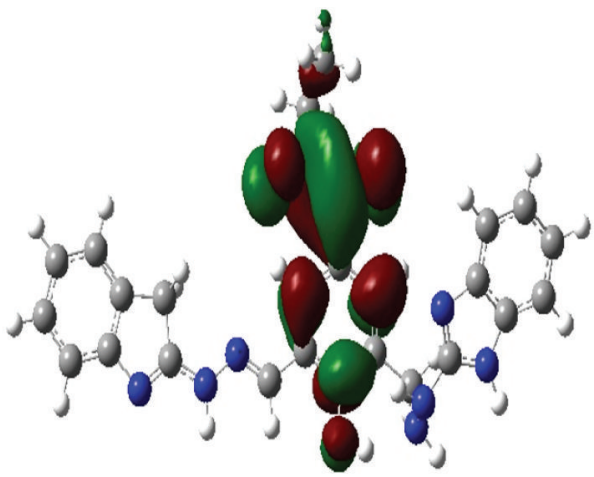

(b)

FIGURE 10: Molecular orbital distribution plots of (a) HOMO and (b) LUMO states in the ground state of Ligand19 at M06/6-31g(d) level in gas phase. 
TABLE 8: Properties of ligand molecules.

\begin{tabular}{lrccccc}
\hline Sl. number & Ligands & Mol.Wt & $\log P$ & Hydrogen bond acceptor & Hydrogen bond donor & Free rotatable bonds \\
\hline 1 & Ligand7 & 821.00 & 4.21 & 15 & 6 & 12 \\
2 & Ligand8 & 499.95 & 3.12 & 11 & 1 & 9 \\
3 & Ligand19 & 495.53 & 3.83 & 10 & 5 & 9 \\
\hline
\end{tabular}

The large hetero atom containing compounds have shown higher $\mathrm{IC}_{50}$ value experimentally in the literature $[46,47]$. Electron donor groups can be identified as having more electron density observed from the HOMO picture of a ligand. In Ligand19 the HOMO is scattered over from phenol to indole group and LUMO is scattered over from phenol to 2-oxo-butanoyl group (Figure 10). The docking results also show that Ligand19 is involved in the important interactions with the key residues of protein. In Figure S7 (at Supplementary Material available online at http://dx.doi.org/10.1155/2016/7264080), for Ligand8, the HOMO is scattered over from the furan to the benzimidazole group and LUMO is scattered only on the 1-chloro-2-nitroimidazole group. In Figure S3, for Ligand7, the HOMO and LUMO are scattered over on the same place of the functional groups. As shown in Figure S11, for Ligand20, the HOMO is scattered from the quinoline group to bromobenzene, but the LUMO is scattered from the azo group to bromobenzene. Qualitatively, in Ligand19 the electron localization between HOMO and LUMO has varied between the functional groups quit higher than the rest of the compounds considered for the DFT study. But, the HOMO-LUMO gap is observed to be smaller in Ligand19 compared to the other ligands. The results of orbital energies are possibly associated with the charge transfer, $\pi \cdots \pi$, or $\pi \cdots \sigma$ stacking between inhibitors and amino acid residues in the binding site of an enzyme.

The electrostatic potential (ESP) structure for Ligand19 is shown in Figure 11. The ESP maps are scaled from $-0.867 *$ $10^{-2}$ to $0.867 * 10^{-2}$. The blue region is observed to be donor and yellow region is observed to be an acceptor. Ligand19 has more electropositive (blue color) and more electronegative (yellow color) units. In Ligand19 the benzimidazole and azo groups are revealed to be a hydrogen bond donor from ESP structure which is consistent with the docking results. The benzimidazole group acts as a donor while interacting with the phenylalanine. Similarly, the nitrogen in indole group acts as a hydrogen bond acceptor which is also consistent with the docking results. The indole group is accepting a proton from the aspartic acid as a hydrogen bond acceptor. The molecular electrostatic potential and orbital energies have the feature of being successfully employed as a 3D structural query for virtual screening of databases to identify the potential inhibitors.

\section{Lipinski Rule of 5}

The screening of compounds has been performed through molecular docking approach. The properties of the ligands are examined using using Qikprop, Schrodinger LLC [48]. The property influences the pharmacological activity derived

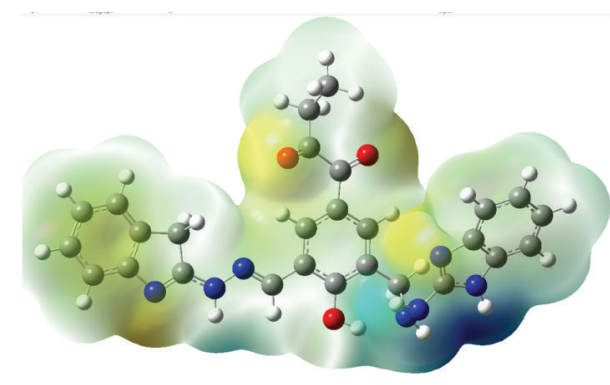

FIGURE 11: Electrostatic potential maps (ESP) of Ligand19 at M06/6$3 \lg (\mathrm{d})$ level in gas phase (scale: $-0.867 * 10^{-2}$ to $8.67 * 10^{-2}$ ).

from the Absorption, Distribution, Metabolism, and Excretion to estimate their drug likeliness of the compound. The binding between the ligand and protein is considered with the number of rotatable bonds; the value was determined as $<10$ which influences the conformational changes [16]. The lowest degree of $\log P$ value indicates the good water solubility according to Lipinski's rule. The property seems to be more significant and forecasts the physicochemical property of the drugs carried out in the part of drug designing [49]. Lipinski rule of 5 (RO5) parameters is satisfied by Ligand8 and Ligand19. In order to consider the obtained ligands with minimal acceptors Ligand19 seems to be chosen as a drug target and is reported in Table 8 .

According to Nguyen et al., compound 2 (here, Ligand8) was the better ligand from biological significance and was assessed using $\mathrm{IC}_{50}$ values through the in vitro and screening studies. Furthermore, the efficiency of structure-based drug designing approach has great impact on investigating the series of compounds. The scoring of molecular mechanics (MMGBSA) based approach strengthens the scoring functions when comparing to the docking analysis. The scoring value has shown the correlation between the computed score and experimental data. It can be considered as reliable approach to handle more structurally dissimilar ligands [9]. In the present study, remarkable results are obtained with this methodology when compared to docking scoring function; the MMGBSA procedure provided more better association between calculated binding free energies and biological activity (Tables 3 and 4), extended to MD simulation and DFT approaches. The study exhibits the significance of the set of inhibitors identified in the order of binding energy, hydrogen bond interactions, gas phase energy values, and physicochemical properties. Ligand19 with the experimental activity of $1.3 \mu \mathrm{M}$ (Table 1 ) has shown the significant activity in terms of hydrogen bond interactions with His51, Asp75, Gly133, Phe130, Gly151, and Gly153 and determines 
the high affinity suggesting a possible mechanism of the action compared to Ligand07 and Ligand08. The current investigation has a good agreement with the earlier reports. Moreover, the molecular modelling approaches enhanced to swift identification and initial optimization of novel series of inhibitors for the NS2B/NS3 proteins.

\section{Conclusions}

In this study, the novel Ligand19 termed as an inhibitor for the dengue virus NS2B/NS3 protease enzyme was interpreted from the series of docking and molecular dynamics simulation protocols. Ligand19 seems to be more potent than Ligand8 and Ligand7 from the outcome of docking, dynamics, DFT, and physicochemical properties analysis. The six residues of the Wild Type (WT) protein were mutated with alanine; the simulation analysis reveals that it may disrupt their stability and leads to changing their conformation. Moreover, the FMO of DFT studies revealed the lowest HOMO-LUMO for Ligand19 reported as a best inhibitor in the study. Our DFT results of binding energy, FMO and ESP, are in good agreement with the docking studies qualitatively and quantitatively. The biological activity of the identified ligand seems to be more significant according to Lipinski's rule of 5 (RO5). The results obtained from this analysis will be sufficient for the further investigations.

\section{Conflict of Interests}

The authors declare that there is no conflict of interests regarding the publication of this paper.

\section{Acknowledgment}

The authors extend their sincere thanks to Dr. D. Velumurugan, Head and Coordinator, BIF, Department of Biophysics and Crystallography, University of Madras, Chennai, India, and to the professor who reviewed this paper.

\section{References}

[1] S. Idrees and U. A. Ashfaq, "A brief review on dengue molecular virology, diagnosis, treatment and prevalence in Pakistan," Genetic Vaccines and Therapy, vol. 10, article 6, 2012.

[2] S. C. Weaver and N. Vasilakis, "Molecular evolution of dengue viruses: contributions of phylogenetics to understanding the history and epidemiology of the preeminent arboviral disease," Infection, Genetics and Evolution, vol. 9, no. 4, pp. 523-540, 2009.

[3] http://www.who.int/mediacentre/factsheets/fs117/en/.

[4] S.-W. Wan, C.-F. Lin, S. Wang et al., "Current progress in dengue vaccines," Journal of Biomedical Science, vol. 20, no. 1, article 37, 2013.

[5] S. Clum, K. E. Ebner, and R. Padmanabhan, "Cotranslational membrane insertion of the serine proteinase precursor NS2B$\mathrm{NS}_{3}$ (Pro) of dengue virus type 2 is required for efficient in vitro processing and is mediated through the hydrophobic regions of NS2B," The Journal of Biological Chemistry, vol. 272, no. 49, pp. 30715-30723, 1997.
[6] E. G. Westaway, J. M. Mackenzie, M. T. Kenney, M. K. Jones, and A. A. Khromykh, "Ultrastructure of Kunjin virus-infected cells: colocalization of NS1 and NS3 with double-stranded RNA, and of NS2B with NS3, in virus-induced membrane structures," Journal of Virology, vol. 71, no. 9, pp. 6650-6661, 1997.

[7] H. Li, S. Clum, S. You, K. E. Ebner, and R. Padmanabhan, “The serine protease and RNA-stimulated nucleoside triphosphatase and RNA helicase functional domains of dengue virus type 2 NS3 converge within a region of 20 amino acids," Journal of Virology, vol. 73, no. 4, pp. 3108-3116, 1999.

[8] L.-R. Jan, C.-S. Yang, D. W. Trent, B. Falgout, and C.-J. Lai, "Processing of Japanese encephalitis virus non-structural proteins: NS2B-NS3 complex and heterologous proteases," Journal of General Virology, vol. 76, no. 3, pp. 573-580, 1995.

[9] R. Khumthong, C. Angsuthanasombat, S. Panyim, and G. Katzenmeier, "In vitro determination of dengue virus type 2 NS2B-NS3 protease activity with fluorescent peptide substrates," Journal of Biochemistry and Molecular Biology, vol. 35, no. 2, pp. 206-212, 2002.

[10] T. T. H. Nguyen, S. Lee, H.-K. Wang et al., "In vitro evaluation of novel inhibitors against the NS2B-NS3 protease of dengue fever virus type 4," Molecules, vol. 18, no. 12, pp. 15600-15612, 2013.

[11] R. I. Brinkworth, D. P. Fairlie, D. Leung, and P. R. Young, "Homology model of the dengue 2 virus NS3 protease: putative interactions with both substrate and NS2B cofactor," Journal of General Virology, vol. 80, no. 5, pp. 1167-1177, 1999.

[12] A. E. Matusan, M. J. Pryor, A. D. Davidson, and P. J. Wright, "Mutagenesis of the Dengue virus type 2 NS3 protein within and outside helicase motifs: effects on enzyme activity and virus replication," Journal of Virology, vol. 75, no. 20, pp. 9633-9643, 2001.

[13] T. J. Chambers, A. Nestorowicz, S. M. Amberg, and C. M. Rice, "Mutagenesis of the yellow fever virus NS2B protein: effects on proteolytic processing, NS2B-NS3 complex formation, and viral replication," Journal of Virology, vol. 67, no. 11, pp. 6797-6807, 1993.

[14] H.-Y. Sun and F.-Q. Ji, "A molecular dynamics investigation on the crizotinib resistance mechanism of C1156Y mutation in ALK," Biochemical and Biophysical Research Communications, vol. 423, no. 2, pp. 319-324, 2012.

[15] Y. Zhao and D. G. Truhlar, "The M06 suite of density functionals for main group thermochemistry, thermochemical kinetics, noncovalent interactions, excited states, and transition elements: two new functionals and systematic testing of four M06-class functionals and 12 other functionals," Theoretical Chemistry Accounts, vol. 120, no. 1-3, pp. 215-241, 2008.

[16] D. F. Veber, S. R. Johnson, H.-Y. Cheng, B. R. Smith, K. W. Ward, and K. D. Kopple, "Molecular properties that influence the oral bioavailability of drug candidates," Journal of Medicinal Chemistry, vol. 45, no. 12, pp. 2615-2623, 2002.

[17] M. J. Frisch, G. W. Trucks, H. B. Schlegel et al., Gaussian 09, Revision A.1, Gaussian, Wallingford, Conn, USA, 2009.

[18] https://pubchem.ncbi.nlm.nih.gov/.

[19] http://www.drugbank.ca.

[20] Chemical Diversity, http://www.chemdiv.com/.

[21] T. Hou, J. Wang, Y. Li, and W. Wang, "Assessing the performance of the MM/PBSA and MM/GBSA methods. 1. The accuracy of binding free energy calculations based on molecular dynamics simulations," Journal of Chemical Information and Modeling, vol. 51, no. 1, pp. 69-82, 2011. 
[22] M. Umamaheswari, A. Madeswaran, and K. Asokkumar, "Virtual screening analysis and in-vitro xanthine oxidase inhibitory activity of some commercially available flavonoids," Iranian Journal of Pharmaceutical Research, vol. 12, no. 3, pp. 317-323, 2013.

[23] I.-J. Chen and N. Foloppe, "Drug-like bioactive structures and conformational coverage with the ligprep/confgen suite: comparison to programs MOE and catalyst," Journal of Chemical Information and Modeling, vol. 50, no. 5, pp. 822-839, 2010.

[24] Protein Databank, http://www.rcsb.org/pdb/home/home.do.

[25] IMPACT, SchrodingerSuite.

[26] R. A. Friesner, J. L. Banks, R. B. Murphy et al., "Glide: a new approach for rapid, accurate docking and scoring. 1. Method and assessment of docking accuracy," Journal of Medicinal Chemistry, vol. 47, no. 7, pp. 1739-1749, 2004.

[27] R. P. C. Valle and B. Falgout, "Mutagenesis of the NS3 protease of dengue virus type 2, Journal of Virology, vol. 72, no. 1, pp. 624-632, 1998.

[28] T. A. Halgren, R. B. Murphy, R. A. Friesner et al., "Glide: a new approach for rapid, accurate docking and scoring. 2. Enrichment factors in database screening," Journal of Medicinal Chemistry, vol. 47, no. 7, pp. 1750-1759, 2004.

[29] R. A. Friesner, R. B. Murphy, M. P. Repasky et al., "Extra precision glide: docking and scoring incorporating a model of hydrophobic enclosure for protein-ligand complexes," Journal of Medicinal Chemistry, vol. 49, no. 21, pp. 6177-6196, 2006.

[30] N. Moitessier, E. Therrien, and S. Hanessian, "A method for induced-fit docking, scoring, and ranking of flexible ligands. Application to peptidic and pseudopeptidic $\beta$-secretase (BACE 1) inhibitors," Journal of Medicinal Chemistry, vol. 49, no. 20, pp. 5885-5894, 2006.

[31] D. Das, Y. Koh, Y. Tojo, A. K. Ghosh, and H. Mitsuya, "Prediction of potency of protease inhibitors using free energy simulations with polarizable quantum mechanics-based ligand charges and a hybrid water model," Journal of Chemical Information and Modeling, vol. 49, no. 12, pp. 2851-2862, 2009.

[32] Y. Koh, D. Das, S. Leschenko et al., "GRL-02031, a novel nonpeptidic protease inhibitor (PI) containing a stereochemically defined fused cyclopentanyltetrahydrofuran potent against multi-pi-resistant human immunodeficiency virus type 1 in vitro," Antimicrobial Agents and Chemotherapy, vol. 53, no. 3, pp. 997-1006, 2009.

[33] U. Essmann, L. Perera, M. L. Berkowitz, T. Darden, H. Lee, and L. G. Pedersen, "A smooth particle mesh Ewald method," The Journal of Chemical Physics, vol. 103, no. 19, pp. 8577-8593, 1995.

[34] H. J. C. Berendsen, J. P. M. Postma, W. F. Van Gunsteren, A. Dinola, and J. R. Haak, "Molecular dynamics with coupling to an external bath," The Journal of Chemical Physics, vol. 81, no. 8, pp. 3684-3690, 1984.

[35] P. Erbel, N. Schiering, A. D’Arcy et al., "Structural basis for the activation of flaviviral NS3 proteases from dengue and West Nile virus," Nature Structural and Molecular Biology, vol. 13, no. 4, pp. 372-373, 2006.

[36] R. Yusof, S. Clum, M. Wetzel, H. M. K. Murthy, and R. Padmanabhan, "Purified NS2B/NS3 serine protease of dengue virus type 2 exhibits cofactor NS2B dependence for cleavage of substrates with dibasic amino acids in vitro," The Journal of Biological Chemistry, vol. 275, no. 14, pp. 9963-9969, 2000.

[37] H. M. Krishna Murthy, K. Judge, L. DeLucas, and R. Padmanabhan, "Crystal structure of dengue virus NS3 protease in complex with a bowman-birk inhibitor: implications for flaviviral polyprotein processing and drug design," Journal of Molecular Biology, vol. 301, no. 4, pp. 759-767, 2000.

[38] L. Y. Kee, T. S. Kiat, H. A. Wahab, R. Yusof, and N. A. Rahman, "Nonsubstrate based inhibitors of dengue virus serine protease: a molecular docking approach to study binding interactions between protease and inhibitors," Asia-Pacific Journal of Molecular Biology and Biotechnology, vol. 15, no. 2, pp. 53-59, 2007.

[39] S. Sanjay and G. R. Desiraju, "N-H...O, O-H...O, and C-H...O hydrogen bonds in protein-ligand complexes: strong and weak interactions in molecular recognition," PROTEINS: Structure, Functions and Bioinformatics, vol. 54, no. 2, pp. 247-259, 2004.

[40] F. Neni, F. C. Chin, M. Z. Sharifuddin, and A. R. Noorsaadah, "Nonsubstrate based inhibitors of dengue virus serine protease: a molecular docking approach to study binding interactions between protease and inhibitors," Asia Pacific Journal of Molecular Biology and Biotechnology, vol. 15, pp. 53-59, 2007.

[41] W. Salaemae, M. Junaid, C. Angsuthanasombat, and G. Katzenmeier, "Structure-guided mutagenesis of active site residues in the dengue virus two-component protease NS2B-NS3," Journal of Biomedical Science, vol. 17, no. 1, pp. 68-75, 2010.

[42] G. Robin, K. Chappell, M. J. Stoermer et al., "Structure of West Nile Virus NS3 protease: Ligand stabilization of the catalytic conformation," Journal of Molecular Biology, vol. 385, no. 5, pp. 1568-1577, 2009.

[43] S. A. Shiryaev, A. E. Aleshin, B. I. Ratnikov, J. W. Smith, R. C. Liddington, and A. Y. Strongin, "Expression and purification of a two-component flaviviral proteinase resistant to autocleavage at the NS2B-NS3 junction region," Protein Expression and Purification, vol. 52, no. 2, pp. 334-339, 2007.

[44] N. H. Mueller, C. Yon, V. K. Ganesh, and R. Padmanabhan, "Characterization of the West Nile virus protease substrate specificity and inhibitors," International Journal of Biochemistry and Cell Biology, vol. 39, no. 3, pp. 606-614, 2007.

[45] P. Niyomrattanakit, P. Winoyanuwattikun, S. Chanprapaph, C. Angsuthanasombat, S. Panyim, and G. Katzenmeier, "Identification of residues in the dengue virus type 2 NS2B cofactor that are critical for NS3 protease activation," Journal of Virology, vol. 78, no. 24, pp. 13708-13716, 2004.

[46] Y. C. Mi, K.-O. Lee, W. K. Jong et al., "Discovery of a novel Her1/Her-2 dual tyrosine kinase inhibitor for the treatment of Her-1 selective inhibitor-resistant non-small cell lung cancer," Journal of Medicinal Chemistry, vol. 52, no. 21, pp. 6880-6888, 2009.

[47] S. Niwata, H. Fukami, M. Sumida et al., "Substituted 3(phenylsulfonyl)-1-phenylimidazolidine-2,4-dione derivatives as novel nonpeptide inhibitors of human heart chymase," Journal of Medicinal Chemistry, vol. 40, no. 14, pp. 2156-2163, 1997.

[48] Qikprop,Schrodinger, USA.

[49] C. A. Lipinski, F. Lombardo, B. W. Dominy, and P. J. Feeney, "Experimental and computational approaches to estimate solubility and permeability in drug discovery and development settings," Advanced Drug Delivery Reviews, vol. 23, no. 1-3, pp. 3-25, 1997. 

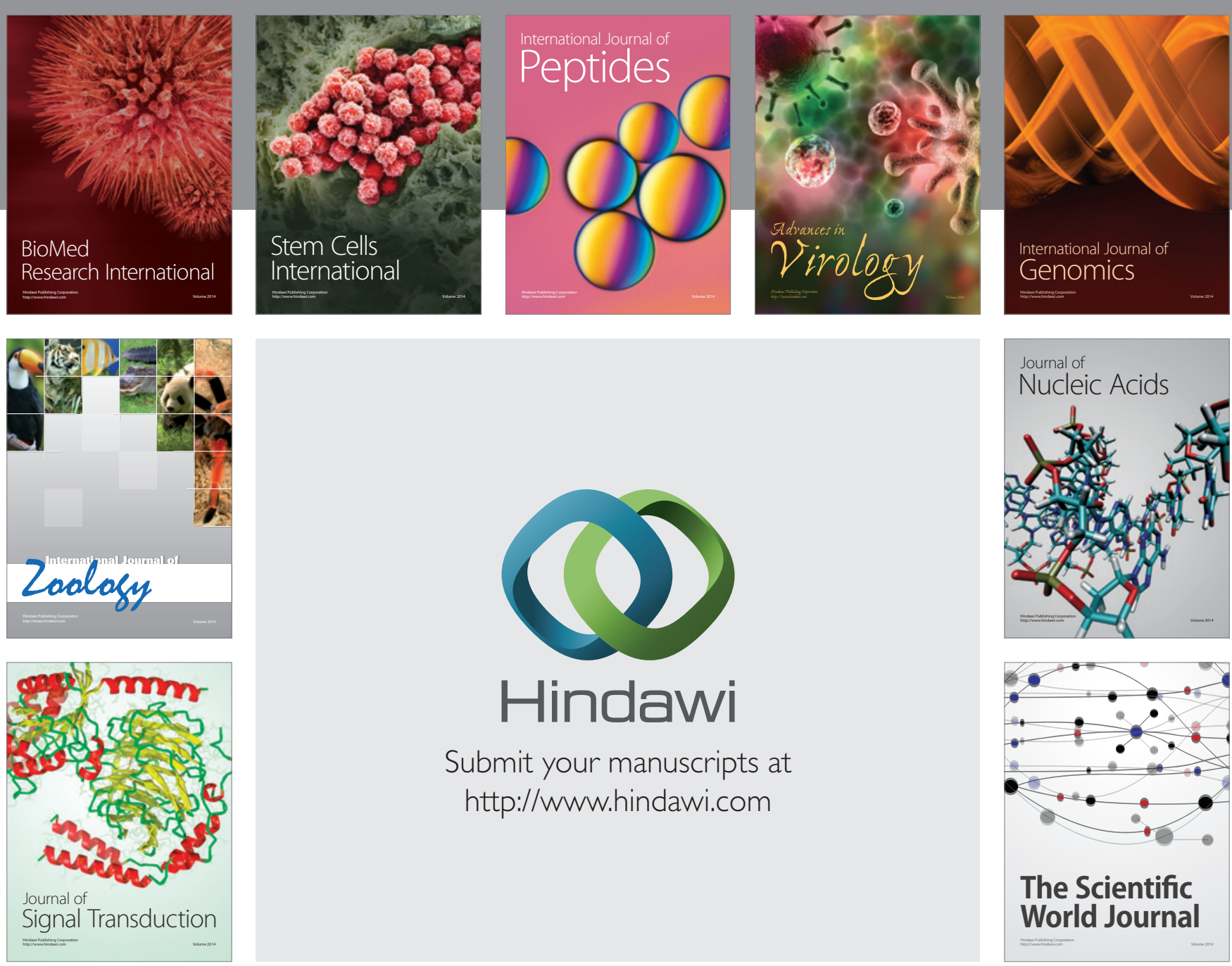

Submit your manuscripts at

http://www.hindawi.com
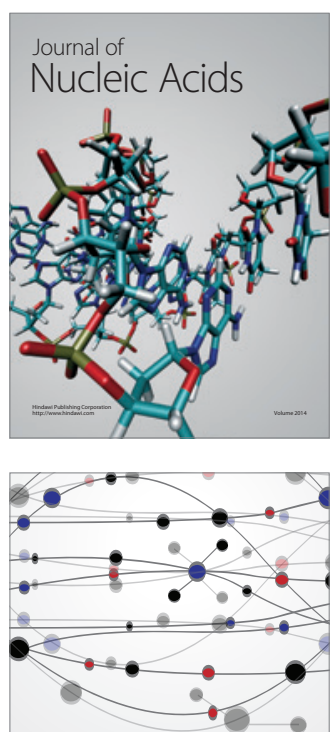

The Scientific World Journal
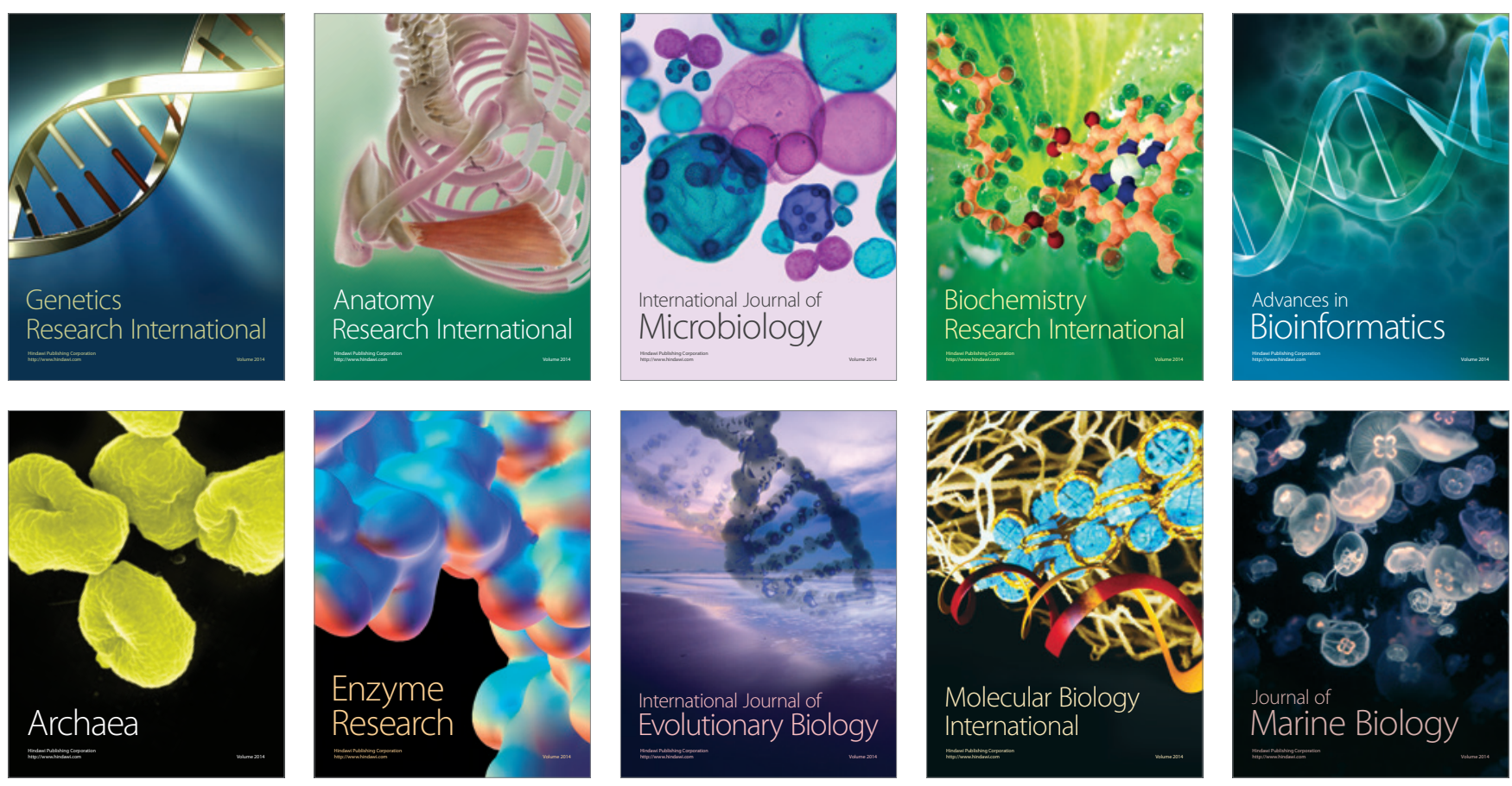